\title{
MODIFICATION OF THE ALGORITHM FOR CALCULATING FATIGUE LIFE FOR THE CRITERIA BASED ON THE CONCEPT OF THE CRITICAL PLANE
}

\author{
Krzysztof Kluger, Tadeusz Łagoda \\ Opole University of Technology, Department of Mechanics and Machine Design, Opole, Poland \\ e-mail:k.kluger@po.opole.pl; t.lagoda@po.opole.pl
}

\begin{abstract}
The aim of this paper is to propose an algorithm for fatigue life determination with the use of widely-known criteria for the fatigue life considering proper determination of material characteristics, which are a function of the number of cycles to failure. The application of the modified algorithm has been presented within the criteria of Findley, Matake, Papadopoulos and Dang Van, and the results of calculations have been compared with test results for steels S355J2G3 and Ck45. For both materials analysed, the application of the modified algorithm in the fatigue criteria makes it possible to obtain much more precise results of the calculations for all types of the loading analysed.
\end{abstract}

Keywords: mean value, bending with torsion, multiaxial fatigue

\section{Introduction}

One of the research areas are the criteria for the multiaxial fatigue life, which aim at the assessment of fatigue damage of the material to any loadings. The fundamental part of the multiaxial fatigue life is the suggested function that reduces the multiaxial state of stress to the uniaxial state. Among many functions suggested, one group features the assumption that the components of the stress state are responsible for fatigue crack initiation associated with the plane at a specified orientation. This suggestion, called the concept of the critical plane, has attracted a lot of attention (Karolczuk and Macha, 2005a,b; Skibicki, 2007; Skibicki and Pejkowski, 2012; Kluger and Łagoda, 2014; Kluger, 2015). The reduction functions, suggested in the criteria, are used to calculate the fatigue life by comparison of the reduced value of stress $\sigma_{e q}$ to stress $\sigma\left(N_{f}\right)$ from a fatigue curve (e.g. of Wöhler or Basquin). The reduction functions based on the critical plane are most often linear or nonlinear functions of material characteristics and the shear stress $\tau_{n s}$, normal stress $\sigma_{n}$ (on the critical plane), or hydrostatic stress $\sigma_{h}$ (stress state invariant). The reduction function properly suggested and applied to any case, but of the same fatigue life, of the uniaxial loading, e.g. torsion, compression, or bending, brings these stress states to the equivalent state. The fatigue criteria in the original form are usually suggested in order to assess the limit state, hence for the so-called fatigue limit. Therefore, the material characteristics are relationships of the fatigue limits from the uniaxial stress states. Unfortunately, the fatigue criteria, or rather reduction functions, applied in order to calculate the so-called limited fatigue life (for steel $N_{f}<2 \cdot 10^{6}$ ), are usually applied with coefficients which are functions of the fatigue limits (Findley et al., 1956; Dang Van et al., 1989; Papadopoulos, 1994; Papuga, 2011; Carpinteri et al., 2013). Such an approach is correct only for materials that have parallel fatigue curves $\left(m_{\sigma} \cong m_{\tau}\right)$. This fact has been noticed in, among others, papers (Kurek and Eagoda, 2012; Karolczuk and Kluger, 2014; Karolczuk et al., 2016).

The aim of this paper is to suggest an algorithm for fatigue life determination with the use of widely-known criteria for the fatigue life considering proper determination of material characteristics which are the functions of the number of cycles to failure. 


\section{Short description of the analysed criteria for multiaxial fatigue life}

\subsection{Findley's criterion}

Findley's criterion (Findley et al., 1956) in the form considering the impact of the mean value of stress has been presented in the following form

$$
\tau_{n s, a}+k \sigma_{n, \max } \leqslant \tau_{a f}
$$

where $k$ is a material constant that takes into account the impact of the normal stress and, according to Findley, depends on the number of cycles to failure, and $\sigma_{\max }=\sigma_{m}+\sigma_{a}$. Findley assumed that the main directions under the proportional loadings do not change. He also drew attention to the fact that the constant $k$ depends on the number of cycles to failure $N_{f}$, and this formula is in the following form for reduction of the stress state according to (2.1)

$$
\frac{\sigma_{f}\left(N_{f}\right)}{\tau_{f}\left(N_{f}\right)}=\frac{2}{1+\frac{k}{\sqrt{1+k^{2}}}}
$$

where $\sigma_{f}\left(N_{f}\right)$ and $\tau_{f}\left(N_{f}\right)$ are fatigue curves for symmetrical bending and symmetrical torsion, respectively.

\subsection{Papadopoulos' criterion}

Papadopoulos (Papadopoulos, 1998) offered a form of the criterion being a linear combination of the maximum amplitude of the generic shear stress $T_{a}$ on the critical plane and the maximum value of the hydrostatic stress $\sigma_{H}$ in the mesoscopic scale, which may be written as

$$
\max T_{a}+k \sigma_{H, \max } \leqslant \tau_{a f}
$$

where $k$ is a coefficient determined by uniaxial fatigue tests in the form of

$$
k=3\left(\frac{\tau_{a f}}{\sigma_{a f}}-\frac{1}{2}\right)
$$

The maximum value of the generic shear stress amplitude of $T_{a}$ is defined as the following

$$
T_{a}(\varphi, \theta)=\sqrt{\frac{1}{\pi} \int_{x=0}^{2 \pi} \tau_{a}^{2}(\varphi, \theta, \chi) d \chi}
$$

where $\tau_{a}$ is the shear stress amplitude in the direction of $\vec{s}$ determined by the angle $x$ on the normal plane $\vec{n}$ defined by angles $\varphi$ and $\theta$ according to the formula

$$
\tau_{a}(\varphi, \theta, \chi)=\frac{1}{2}\left[\max _{t} \tau(\varphi, \theta, \chi, t)-\min _{t} \tau(\varphi, \theta, \chi, t)\right]
$$

\subsection{Matake's criterion}

Matake (Matake, 1977) simplified criterion (2.1) by changing the definition of the critical plane. According to Matake, the critical plane is the maximum shear stress plane

$$
\tau_{n s, a}+k \sigma_{n, \max } \leqslant \tau_{a f}
$$

Under this approach, there is only one coefficient in the criterion, which is determined using the following formula

$$
k=2 \frac{\tau_{a f}}{\sigma_{a f}}-1
$$




\subsection{Dang Van's criterion}

The Dang Van's criterion (Dang Van, 1983; Dang Van et al., 1989) is based on the analysis of stress in the mesoscopic scale, which differs from the stress in the macroscopic scale with "the deviatoric part of the stabilized residual stress tensor". The Dang Van's criterion in the macroscopic scale has been applied in this paper

$$
\tau_{n s}+k \sigma_{H, \max } \leqslant \tau_{a f} \quad k=3 \frac{\tau_{a f}}{\sigma_{a f}}-\frac{3}{2}
$$

\section{Enhanced algorithm for calculating the fatigue life}

The algorithm for calculating the number of cycles to failure $N_{\text {cal }}$ for both proportional and non-proportional loadings considering the variability of the coefficient $k$ according to the number of cycles $N$ is presented in Fig. 1 (Karolczuk et al., 2016).

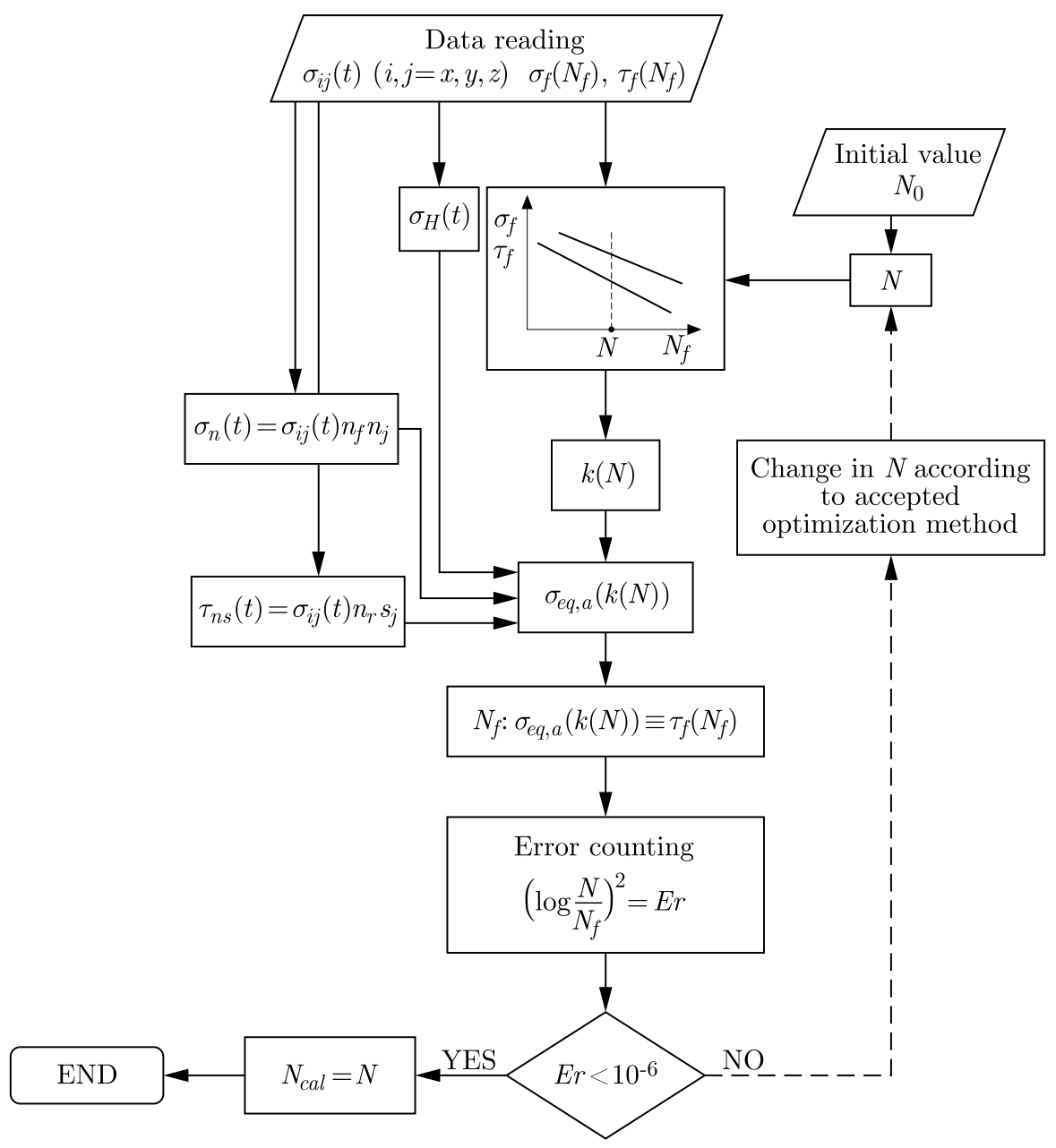

Fig. 1. Algorithm for calculating the fatigue life for proportional loadings considering the variability of the coefficient $k$ according to the number of cycles $N$

In the first phase, the loading of stress state amplitudes $\sigma_{i j, a}$ takes place for the global coordination system $O x y z$ and for material constants that define two fatigue curves: $\sigma_{f}\left(N_{f}\right)$ and $\tau_{f}\left(N_{f}\right)$. Additionally, a set of unit vectors $\mathbf{n}$ normal to the analysed plane is generated, among which the critical plane orientation is sought. On the basis of $\sigma_{i j, a}$ and for each vector $\mathbf{n}$, the vector of stress $\mathbf{T}=\boldsymbol{\sigma} \cdot \mathbf{n}$ is calculated by the inner product of the tensor $\boldsymbol{\sigma}$ and vector $\mathbf{n}$. The 
normal stress amplitude $\sigma_{n, a}$ is calculated by the inner product of the vector $\mathbf{T}$ and vector $\mathbf{n}$. The shear stress amplitude $\tau_{n s, a}$ results from adding the normal and shear stress vectors, that is $\tau_{n s, a}(\mathbf{n})=\sqrt{\mathbf{T}} \cdot \mathbf{T}-\sigma_{n, a}^{2}$. In the second path of the algorithm, looped and optimised for the assumed number of cycles $N$, the value of the coefficient $k$ is calculated on the basis of the assumed fatigue curves $\sigma_{f}\left(N_{f}\right)$ and $\tau_{f}\left(N_{f}\right)$. In the next phase, a set of equivalent stress amplitudes $\sigma_{e q, a}$ (in set $\mathbf{n}$ ) for the assumed $N$ is calculated. Then the set $\sigma_{e q, a}(\mathbf{n})$ is browsed in order to determine the orientation $\mathbf{n}_{c}$ of the critical plane according to the criterion adopted. The determined value $\sigma_{e q, a}\left(\mathbf{n}_{c}\right)$ is substituted to fatigue curves $\sigma_{f}\left(N_{f}\right)$ or $\tau_{f}\left(N_{f}\right)$ to determine a possible number of cycles to failure $N_{f}$. With the use of the objective function

$$
\left(\log \frac{N}{N_{f}}\right)^{2}=E r
$$

the concurrence of the number of cycles $N$ and $N_{f}$ is determined. If the error $E r$ is less than $10^{-6}$, the algorithm is terminated by adopting $N_{c a l}=N$. In another case, the value $N$ is altered and the second path of the algorithm is repeated.

\section{Experimental data}

The suggested algorithm has been verified on the basis of the experimental data concerning steels S355J2G3 (Pawliczek and Prażmowski, 2015; Kluger and Łagoda, 2014) and Ck45, which were taken from the literature (Simbürger, 1975).

Based on the data, the coefficients of fatigue curves have been calculated under uniaxial loadings according to the ASTM standards (ASTM E1049-85(2011)e1, 2003):

— for bending

$$
\sigma_{f}\left(N_{f}\right): \quad \log N_{f}=A_{\sigma}-m_{\sigma} \log \sigma_{f}
$$

- for torsion

$$
\tau_{f}\left(N_{f}\right): \quad \log N_{f}=A_{\tau}-m_{\tau} \log \tau_{f}
$$

where $N_{f}$ is the estimated number of cycles to failure, $A_{\sigma}, m_{\sigma}, A_{\tau}, m_{\tau}$ are parameters of the linear regression equation. The basic strength and fatigue parameters of the materials analysed are presented in Table 1. Types of analysed loadings for steel S355J2G3 and Ck45 are presented in Table 2 .

Table 1. The basic strength parameters and material constants for fatigue curves (4.1) and (4.2) with the confidence intervals for a probability of 0.95

\begin{tabular}{|l|c|c|c|c|c|c|c|c|}
\hline $\begin{array}{c}\text { Material } \\
(\mathrm{EN})\end{array}$ & $\begin{array}{c}E \\
{[\mathrm{GPa}]}\end{array}$ & $\begin{array}{c}R_{e} \\
{[\mathrm{MPa}]}\end{array}$ & $\begin{array}{c}R_{m} \\
{[\mathrm{MPa}]}\end{array}$ & $\nu$ & $A_{\sigma}$ & $m_{\sigma}$ & $A_{\tau}$ & $m_{\tau}$ \\
\hline \hline S355J2G3 & 213 & 394 & 611 & 0.31 & $23.8 \pm 4.0$ & $7.2 \pm 1.6$ & $32.8 \pm 8.7$ & $11.7 \pm 3.8$ \\
\hline $\mathrm{Ck} 45$ & 210 & 704 & 850 & 0.30 & $28.5 \pm 3.9$ & $9.0 \pm 1.5$ & $77.0 \pm 13.5$ & $29.3 \pm 5.5$ \\
\hline
\end{tabular}

The evaluation of the effectiveness of criteria for the multiaxial fatigue of materials for a limited number of cycles to failure typically involves comparison of the calculated strength $N_{\text {cal }}$ with the experimental one $N_{\text {exp }}$ on a log-log diagram with additionally calculated parameters of the scatter of results (Karolczuk and Kluger, 2014; Kluger, 2015). In order to assess the effectiveness of the algorithm suggested, an original function $\operatorname{Pr}(T)$ has been proposed

$$
\operatorname{Pr}(T)=\operatorname{Prob}\left(\frac{N_{\text {exp }}}{T} \leqslant N_{c a l} \leqslant T N_{\text {exp }}\right) \quad \text { for } \quad T \geqslant 1
$$


Table 2. Types of the analysed loading for steel S355J2G3 and Ck45

\begin{tabular}{|l|l|}
\hline \multicolumn{1}{|c|}{ S355J2G3 } & \multicolumn{1}{c|}{ Ck45 } \\
\hline \hline \multicolumn{2}{|c|}{ Zero mean stress } \\
\hline$\sigma_{a} \neq 0$ & $\sigma_{a} \neq 0$ \\
\hline$\tau_{a} \neq 0$ & $\tau_{a} \neq 0$ \\
\hline$\sigma_{a}=\tau_{a} \neq 0, \varphi=0^{\circ}$ & $\sigma_{a}=\tau_{a} \neq 0, \varphi=0^{\circ}$ \\
\hline$\sigma_{a}=2 \tau_{a} \neq 0, \varphi=90^{\circ}$ & $\sigma_{a}=1.7 \tau_{a}, \varphi=60^{\circ}$ \\
\hline$\sigma_{a}=3 \tau_{a} \neq 0, \varphi=90^{\circ}$ & $\sigma_{a}=1.7 \tau_{a}, \varphi=90^{\circ}$ \\
\hline \multicolumn{2}{|c|}{ Non-zero mean stress } \\
\hline$\sigma_{a} \neq 0, \sigma_{m} \neq 0 \quad$ & $\sigma_{a} \neq 0, \sigma_{m} \neq 0$ \\
\hline$\tau_{a} \neq 0, \tau_{m} \neq 0$ & $\sigma_{a}=1.7 \tau_{a}, \tau_{m} \neq 0, \varphi=0^{\circ}$ \\
\hline$\sigma_{a}=\tau_{a} \neq 0, \sigma_{m}=\tau_{m} \neq 0, \varphi=0^{\circ}$ & $\sigma_{a}=1.7 \tau_{a}, \sigma_{m} \neq 0, \varphi=0^{\circ}$ \\
\hline \multicolumn{2}{|c|}{} \\
\hline
\end{tabular}

and

$$
T(\operatorname{Pr})=0.95
$$

Function (4.3) describes the probability that the calculated fatigue life $N_{c a l}$ is within the scatter band with the coefficient of $T$, wherein $T \geqslant 1$, which means that $T=N_{\text {cal }} / N_{\text {exp }}$ for $N_{\text {cal }} \geqslant N_{\text {exp }}$ or $T=N_{\text {exp }} / N_{\text {cal }}$ for $N_{c a l}<N_{\text {exp }}$. This is an increasing function, based on which one can estimate (through interpolation) the scatter band $T$, which includes, for example, $95 \%$ of the specimens, see equation (4.4).

\section{Results of calculations and their analysis}

The number of cycles to failure $N_{\text {cal }}$ has been calculated with the use of two algorithms, which, for the sake of their presentation clarity, have been marked as: $N A$ - the new algorithm suggested in this paper that takes into account the variability of the coefficient $k$ according to the number of cycles $N ; C A$ - the classical algorithm in which the coefficient $k$ is constant and corresponds to the theoretical limit fatigue, i.e. for $N_{f}=2 \cdot 10^{6}$ cycles. Figures 2 to 9 show a comparison of the experimental number of cycles $N_{\exp }$ with the calculated number of cycles to failure. Additionally, each of these figures features dispersion parameters $T(0.95)$ calculated for each type of the loading. The dotted line represents the scatter band for the coefficient equal to 3 , and the solid line represents the ideal correspondence. In the case of the parameter $T$ exceeding the value of 50, the precise value of $T$ has not been given since such a high value of dispersion renders the given approach unusable. The scatter band $T(0.95)$ is calculated separately for each type of the loading ( $\sigma_{a}$ - bending, $\tau_{a}$ - torsion, $\sigma_{a}-\tau_{a}$-proportional bending-torsion and $\sigma_{a^{-}} \tau_{a^{-}}$ non-proportional bending-torsion, etc.) and also for the results from all types of the loading treated as a set (a total scatter band). In such a case, not all points $N_{\text {exp }}-N_{\text {cal }}$ are within the boundaries of graphs in the figure. Such a scattering is due to the imperfections of the analyzed fatigue criteria for some types of the load.

In order to estimate the level of improvement, a proportion between the scatter bands $T(N A) / T(C A)$ is calculated for computations with the use of both the new and classical algorithms. The correlation improvement is achieved for $T(N A) / T(C A)<1$. The results of the comparison of the scatter bands are presented in Fig. 10.

The graphical comparison of the results obtained with the use of selected comparative fatigue criteria with the experimental results, which are presented in Figs, 2 to 9, has enabled a more 

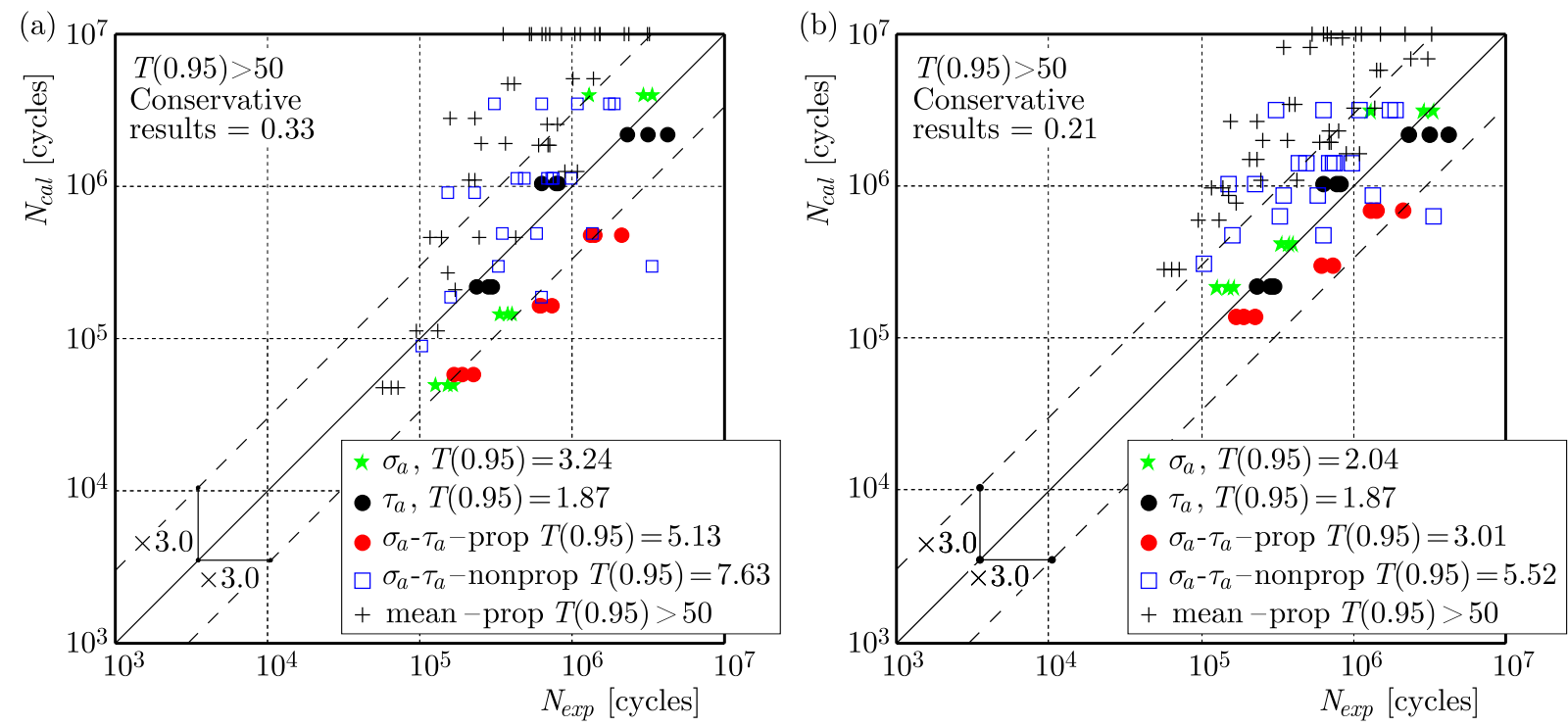

Fig. 2. Comparison of the experimental fatigue life $N_{\text {exp }}$ with the calculated fatigue life $N_{\text {cal }}$ for the Findley criterion and for S355J2G3 steel, according to: (a) classical algorithm $C A$,

(b) new algorithm $N A$
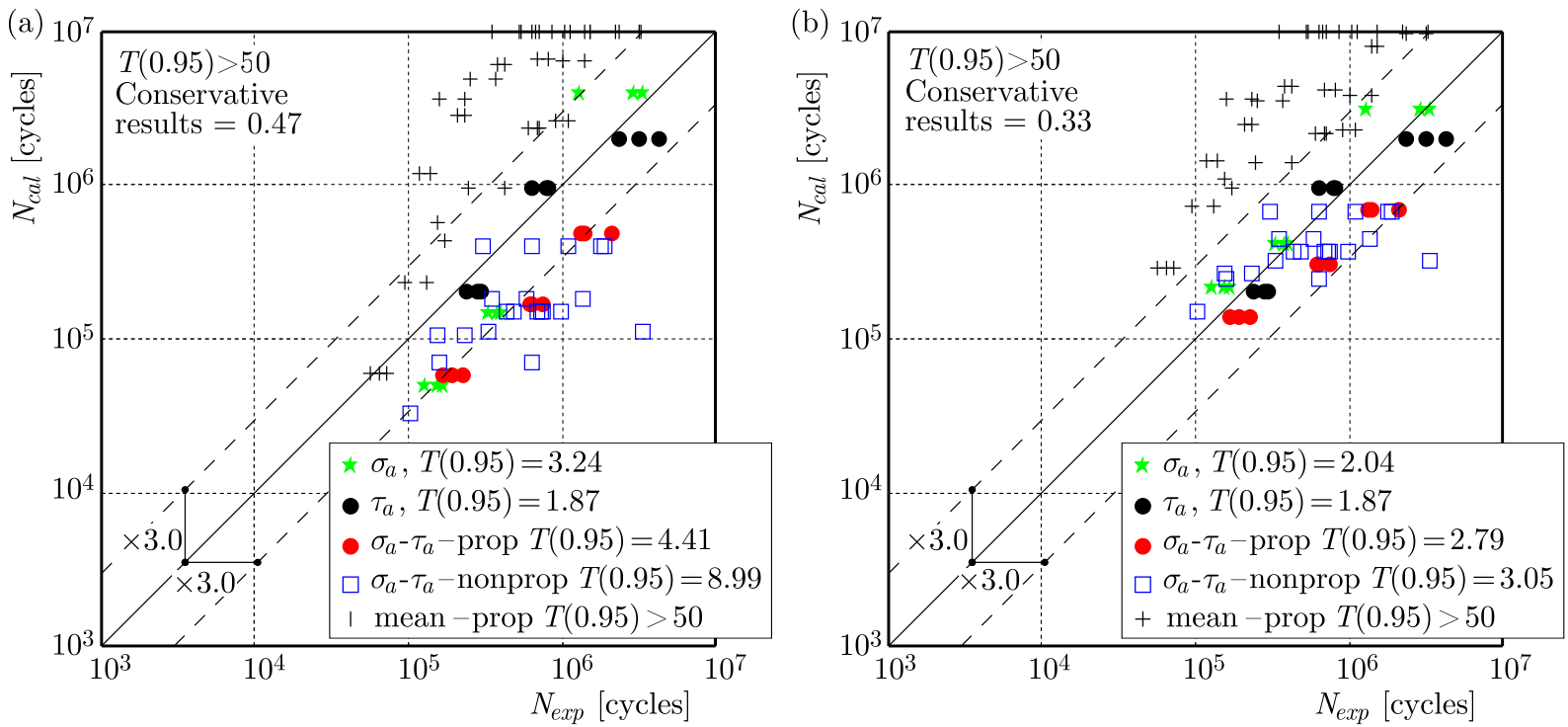

Fig. 3. Comparison of the experimental fatigue life $N_{\text {exp }}$ with the calculated fatigue life $N_{\text {cal }}$ for the Papadopoulos criterion and for S355J2G3 steel, according to: (a) classical algorithm $C A$,

(b) new algorithm $N A$

profound assessment of the capacity of applying the algorithms being described in the fatigue criteria, and also given insight into their usefulness for the estimation of the fatigue life of selected construction materials. A considerable increase in the calculation results has been achieved for all materials analysed and for most types of the loading.

The higher scatter bands achieved for loadings in the presence of nominal stress is caused by an insufficient consideration of the nominal stress in the fatigue criteria. 

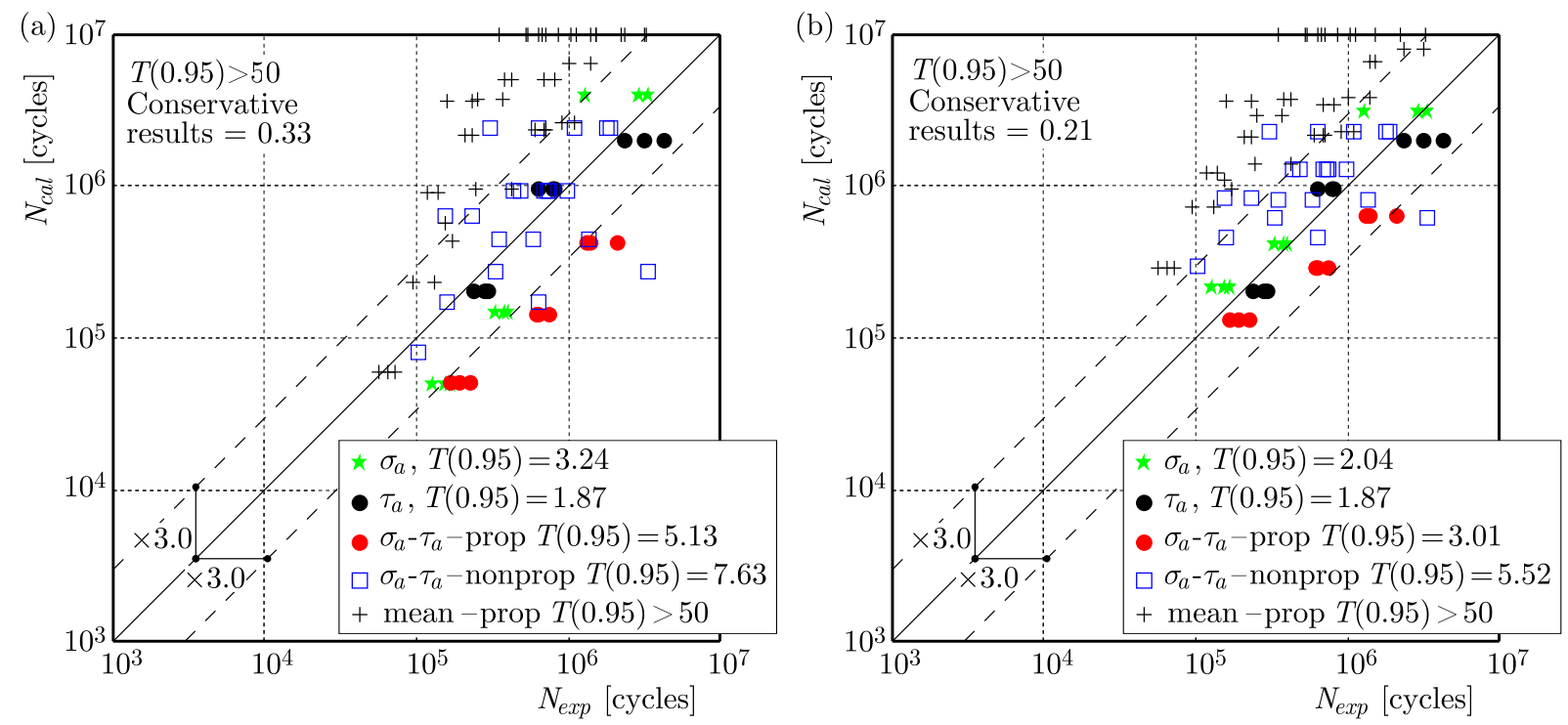

Fig. 4. Comparison of the experimental fatigue life $N_{\exp }$ with the calculated fatigue life $N_{c a l}$ for the Matake criterion and for S355J2G3 steel, according to: (a) classical algorithm $C A$,

(b) new algorithm $N A$
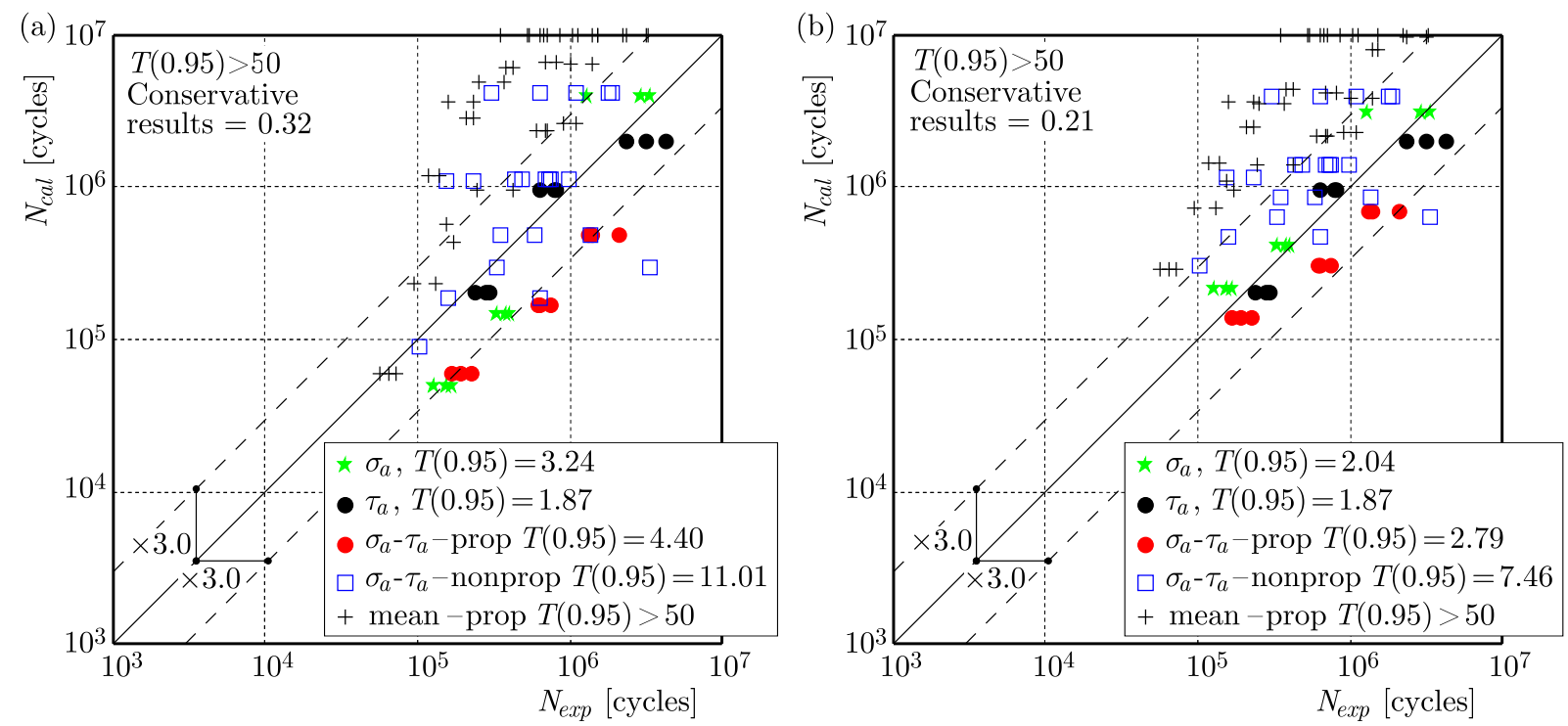

Fig. 5. Comparison of the experimental fatigue life $N_{\text {exp }}$ with the calculated fatigue life $N_{\text {cal }}$ for the

Dang Van criterion and for S355J2G3 steel, according to: (a) classical algorithm $C A$,

(b) new algorithm $N A$

\section{Conclusions}

On the basis of the analyses performed, the following conclusions may be drawn:

- The suggested algorithm for calculating the fatigue life that takes into account the variability of the coefficients occurring in the fatigue criteria according to a number of cycles is concurrent in the analysed proportional ranges of proportional and non-proportional cyclic loadings, with non-zero mean stress, both in the presence and absence of the nominal stress. 

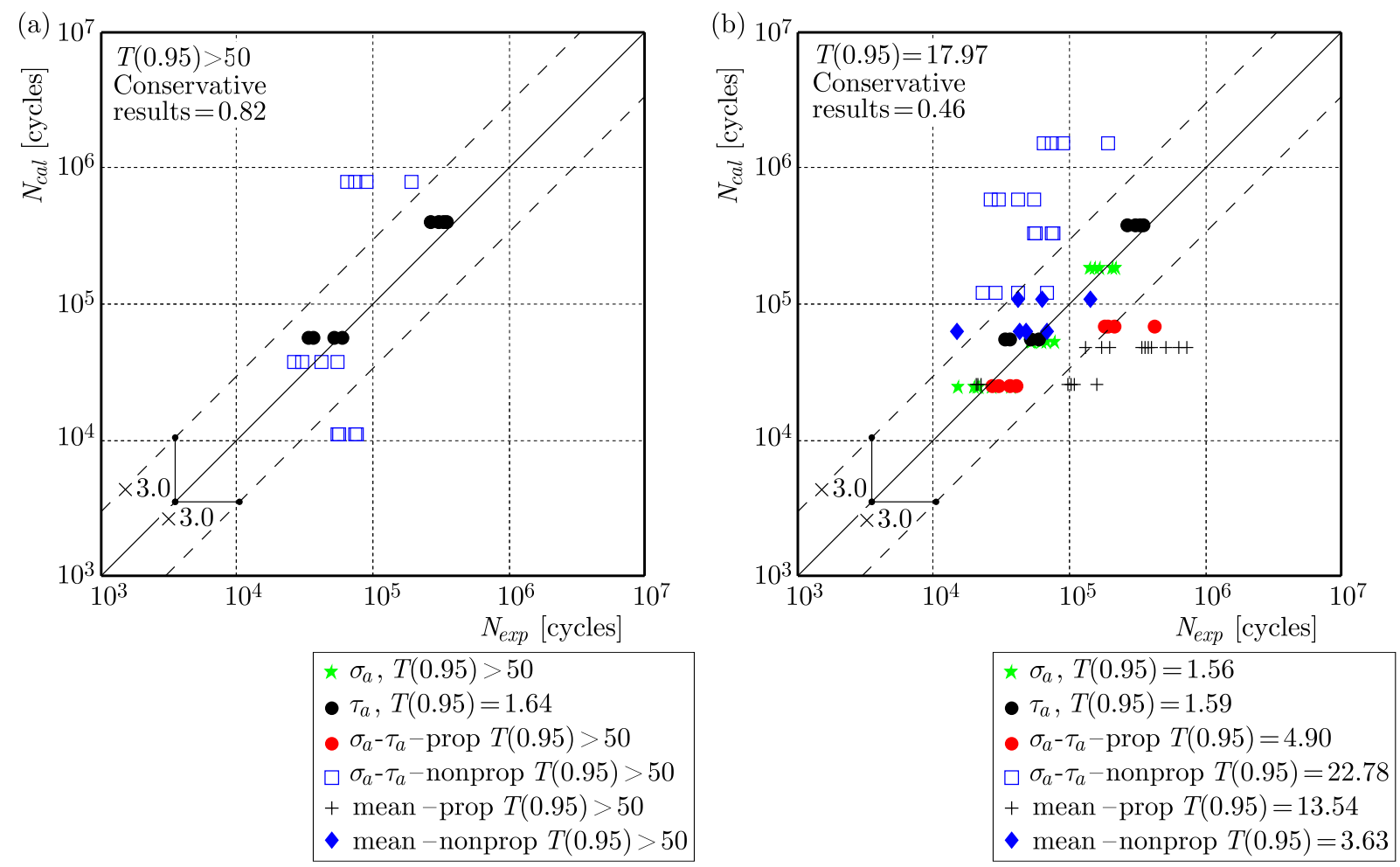

Fig. 6. Comparison of the experimental fatigue life $N_{\text {exp }}$ with the calculated fatigue life $N_{c a l}$ for the Findley criterion and for Ck45 steel, according to: (a) classical algorithm $C A$, (b) new algorithm $N A$
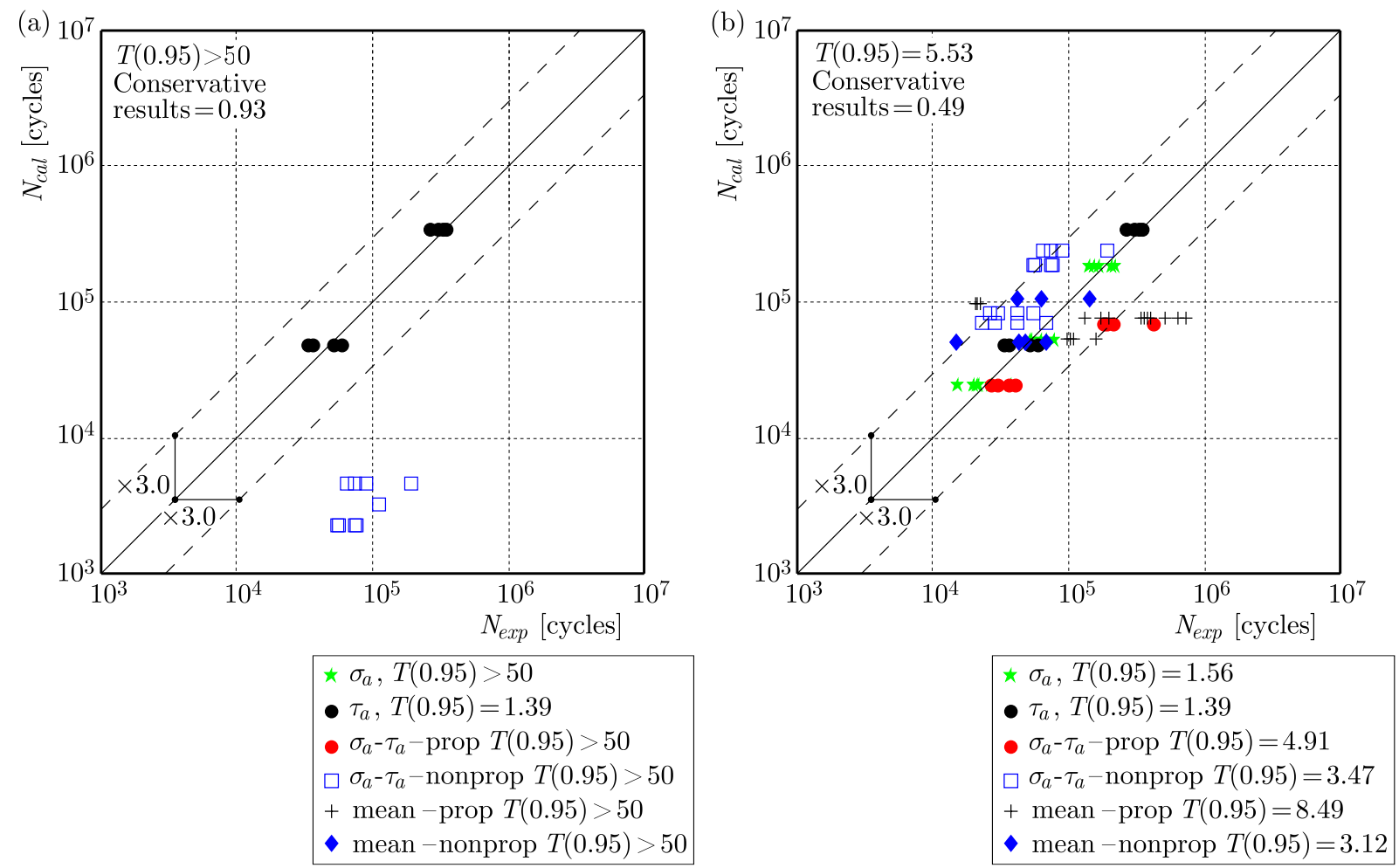

Fig. 7. Comparison of the experimental fatigue life $N_{\text {exp }}$ with the calculated fatigue life $N_{c a l}$ for the Papadopoulos criterion and for Ck45 steel, according to: (a) classical algorithm $C A$,

(b) new algorithm $N A$ 


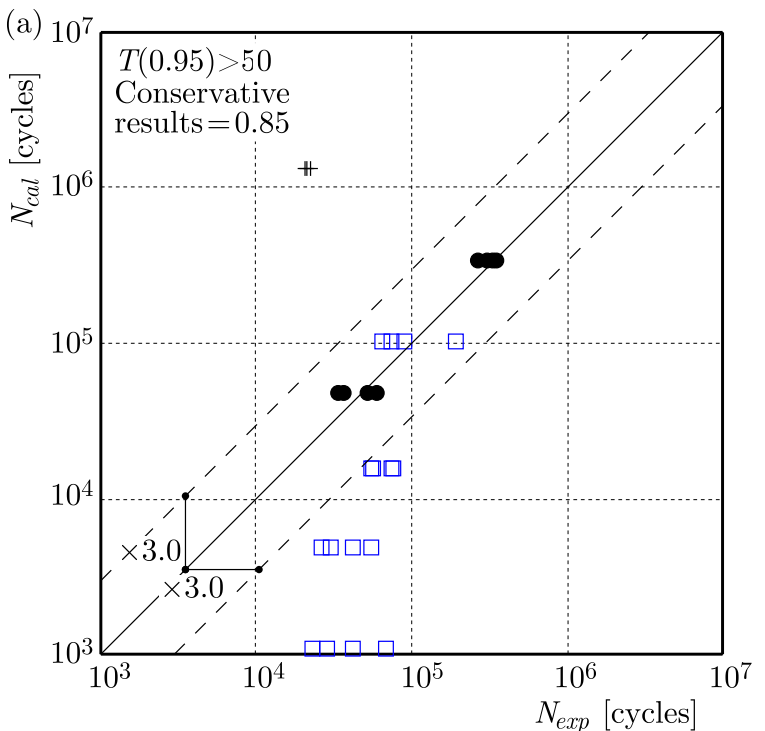

$$
\begin{aligned}
& \sigma_{a}, T(0.95)>50 \\
& \text { - } \tau_{a}, T(0.95)=1.39 \\
& \text { - } \sigma_{a}-\tau_{a} \text {-prop } T(0.95)>50 \\
& \square \sigma_{a}-\tau_{a}-\text { nonprop } T(0.95)=42.89 \\
& + \text { mean - prop } T(0.95)>50 \\
& \text { - mean - nonprop } T(0.95)>50
\end{aligned}
$$

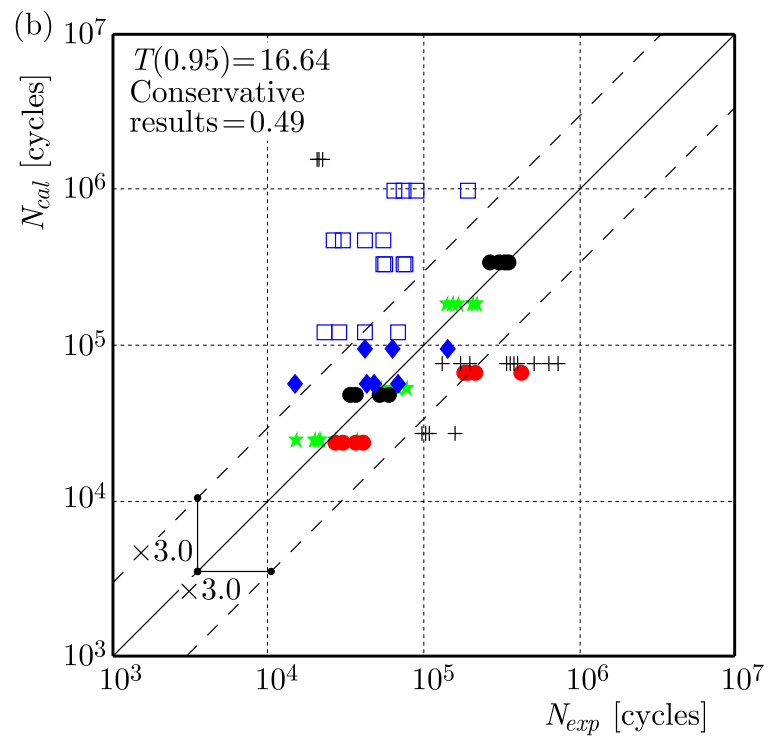

$$
\begin{aligned}
& \star \sigma_{a}, T(0.95)=1.56 \\
& \text { - } \tau_{a}, T(0.95)=1.39 \\
& \text { - } \sigma_{a}-\tau_{a} \text {-prop } T(0.95)=5.10 \\
& \square \sigma_{a}-\tau_{a}-\text { nonprop } T(0.95)=16.15 \\
& + \text { mean - prop } T(0.95)>50 \\
& \checkmark \text { mean -nonprop } T(0.95)=3.24
\end{aligned}
$$

Fig. 8. Comparison of the experimental fatigue life $N_{\exp }$ with the calculated fatigue life $N_{\text {cal }}$ for the Matake criterion and for $\mathrm{Ck} 45$ steel, according to: (a) classical algorithm $C A$, (b) new algorithm $N A$

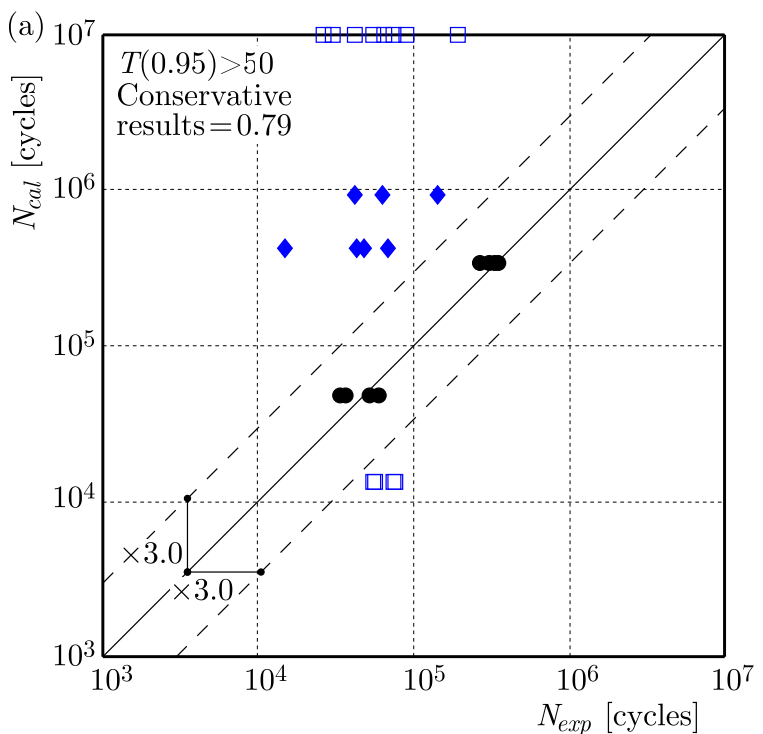

$$
\star \sigma_{a}, T(0.95)>50
$$$$
\text { - } \tau_{a}, T(0.95)=1.39
$$$$
\text { - } \sigma_{a}-\tau_{a} \text {-prop } T(0.95)>50
$$$$
\square \sigma_{a}-\tau_{a}-\text { nonprop } T(0.95)>50
$$$$
+ \text { mean - prop } T(0.95)>50
$$$$
\text { - mean -nonprop } T(0.95)=26.15
$$

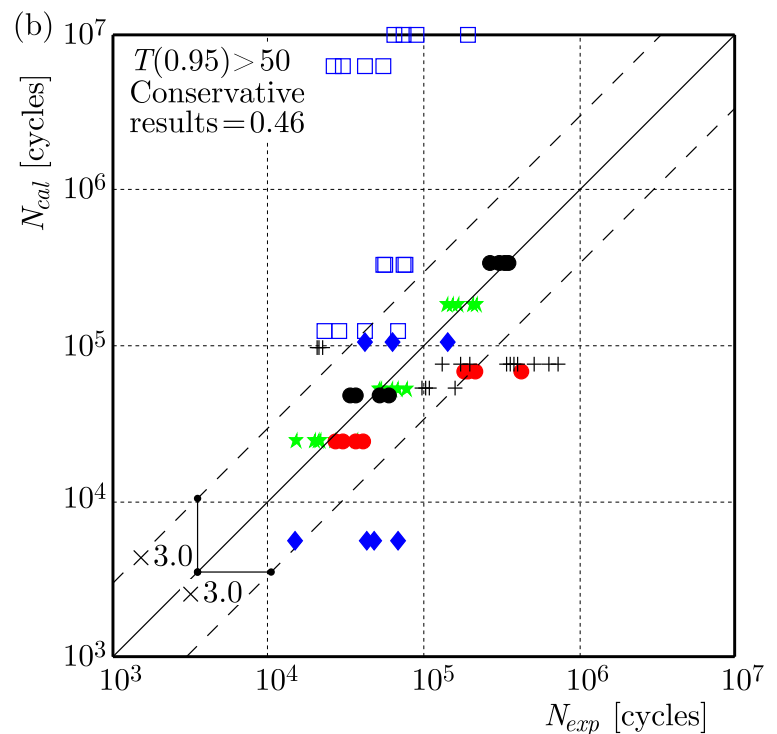

$\star \sigma_{a}, T(0.95)=1.56$

- $\tau_{a}, T(0.95)=1.39$

- $\sigma_{a}-\tau_{a}-$ prop $T(0.95)=4.91$

$\square \sigma_{a}-\tau_{a}-$ nonprop $T(0.95)>50$

+ mean - prop $T(0.95)=8.49$

- mean -nonprop $T(0.95)=10.89$

Fig. 9. Comparison of the experimental fatigue life $N_{\text {exp }}$ with the calculated fatigue life $N_{\text {cal }}$ for the Dang Van criterion and for Ck45steel, according to: (a) classical algorithm $C A$, (b) new algorithm $N A$ 

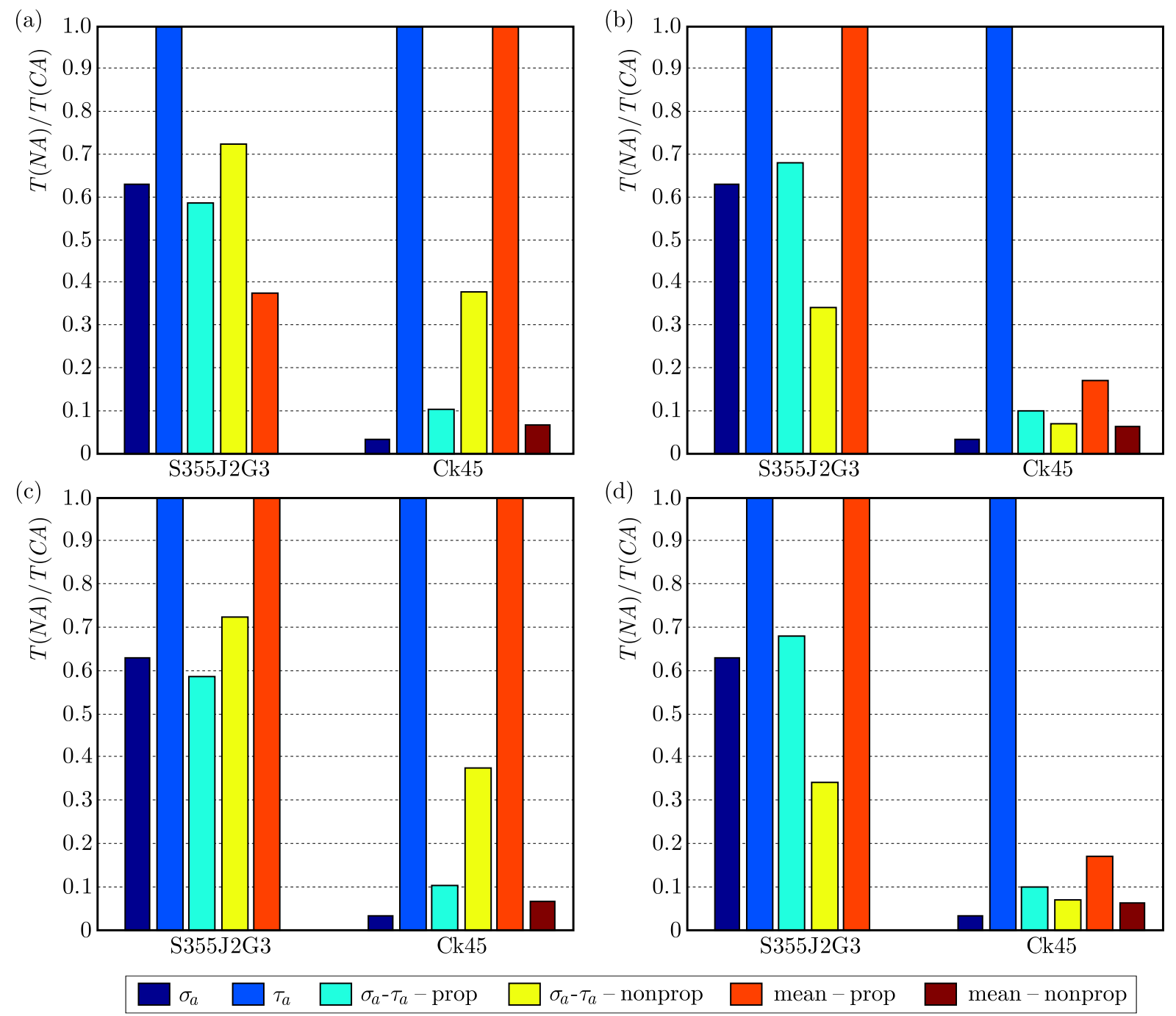

Fig. 10. Comparison of the scatter bands of the new and classical algorithms for the materials and types of the loading analysed: (a) Findley criterion, (b) Papadopoulos criterion, (c) Matake criterion,

(d) Dang Van criterion

- By the variability of the coefficients used in the fatigue criteria according to the number of cycles, a considerable increase in the correspondence between the calculation and experimental results for steel S355J2G3 and $\mathrm{Ck} 45$ has been achieved.

- The discrepancies in the results of the experiment and calculations in the presence of the nominal stress are caused by neglecting the shear stress in the criteria analysed.

- The modified algorithm described here may be applied to most criteria in the literature that are based on the concept of the critical plane.

\section{References}

1. ASTM E1049-85(2011)e1, 2003, Standard Practices for Cycle Counting in Fatigue Analysis, ASTM International, West Conshohocken, PA

2. Carpinteri A., Spagnoli A., Vantadori S., Bagni C., 2013, Structural integrity assessment of metallic components under multiaxial fatigue: the C-S criterion and its evolution, Fatigue and Fracture of Engineering Materials and Structures, 36, 9, 870-883 
3. Dang VAn K., 1983, Macro-micro approach in high-cycle multiaxial fatigue, American Society for Testing and Materials STP 1191, 120-130

4. Dang Van K., Cailletaud G., Flavenot J.F., Le Douaron A., Lieurade H.P., 1989, Criterion for high cycle fatigue failure under multiaxial loading, Mechanical Engineering Publications, London, 459-478

5. Findley W.N., Coleman J.J., Hanley B.C., 1956. Theory for combined bending and torsion fatigue with data for SAE 4340 steel, International Conference on Fatigue of Metals, London

6. Karolczuk A., Kluger K., 2014, Analysis of the coefficient of normal stress effect in chosen multiaxial fatigue criteria, Theoretical and Applied Fracture Mechanics, 73, 39-47

7. Karolczuk A., Kluger K., Łagoda T., 2016, A correction in the algorithm of fatigue life calculation based on the critical plane approach, International Journal of Fatigue, 83, 174-183

8. Karolczuk A., Macha E., 2005a, Critical planes in multiaxial fatigue, [In:] Materials Structure and Micromechanics of Fracture, J. Pokluda (Edit.), Zurich-Uetikon: Trans Tech Publications Ltd, 109-114

9. Karolczuk A., Macha E., 2005b. Fatigue fracture planes and expected principal stress directions under biaxial variable amplitude loading, Fatigue Fracture of Engineering Materials and Structures, 28, 1/2, 99-106

10. Kluger K., 2015, Fatigue life estimation for 2017A-T4 and 6082-T6 aluminium alloys subjected to bending-torsion with mean stress, International Journal of Fatigue, 80, 22-29

11. Kluger K., Łagoda T., 2014, New energy model for fatigue life determination under multiaxial loading with different mean values, International Journal of Fatigue, 66, 229-245

12. Kurek M., EAgoda T., 2012, Estimation of fatigue life of materials with out-of-parallel fatigue characteristics under block loading, Materials Science Forum, 726, 181-188

13. Matake T., 1977, An explanation on fatigue limit under combined stress, Bulletin of the JSME, 20, 141, 257-263

14. Papadopoulos I.V., 1994, A new criterion of fatigue-strength for out-of-phase bending and torsion, International Journal of Fatigue, 16, 6, 377-384

15. Papadopoulos I.V., 1998, Critical plane approaches in high-cycle fatigue: on the definition of the amplitude and mean value of the shear stress acting on the critical plane, Fatigue and Fracture of Engineering Materials and Structures, 21, 3, 269-285

16. Papuga J., 2011, A survey on evaluating the fatigue limit under multiaxial loading, International Journal of Fatigue, 33, 2, 153-165

17. Pawliczek R., Prażmowski M., 2015, Study on material property changes of mild steel S355 caused by block loads with varying mean stress, International Journal of Fatigue, 80, 171-177

18. Simbürger A., 1975, Festigkeitsverhalten zäher Werkstoffe bei einer mehrachsigen phasenverschobenen Schwingbeanspruchung mit körperfesten und veränderlichen Hauptspannungsrichtun-gen. LBF Darmstadt - Bericht Nr FB-1975.

19. SkiBiCKi D., 2007, Experimental verification of fatigue loading nonproportionality model, Journal of Theoretical and Applied Mechanics, 45, 2, 337-348

20. Skibicki D., Pejkowski L., 2012, Integral fatigue criteria evaluation for life estimation under uniaxial combined proportional and non-proportional loadings, Journal of Theoretical and Applied Mechanics, 50, 4, 1073-1086 